\title{
A new artificial root system to simulate the effects of transpiration-induced suction and root reinforcement
}

\author{
V. Kamchoom ${ }^{\text {i) }}$, A. K. Leung ${ }^{\text {ii) }}$ and C.W.W. Ng ${ }^{\text {iii }}$ \\ i) Ph.D. Student, Department of Civil and Environmental Engineering, Hong Kong University of Science and Technology, Hong Kong. \\ ii) Lecturer, Division of Civil Engineering, University of Dundee, Dundee, UK. \\ iii) Chair Professor, Department of Civil and Environmental Engineering, Hong Kong University of Science and Technology, Hong \\ Kong.
}

\begin{abstract}
Vegetation is increasingly recognised as an eco-friendly method for slope stabilisation, through mechanical root reinforcement and transpiration-induced soil suction. Several centrifuge tests have been carried out to investigate failure mechanism of slopes mechanically reinforced by roots. However, the effects of transpiration-induced soil suction were ignored, and hence any of its contribution to stability enhancement is not well-understood. This paper aims to develop and verify a new artificial root that is capable of simulating both the effects of transpiration-induced suction and mechanical reinforcement for physical modelling, including centrifuge at high-g. The artificial root was made of a high air-entry value (AEV) porous filter and supplied with vacuum pressures. The function of the porous filter is to maintain hydraulic gradient between soil and the system. Any reduction of soil moisture due to applied vacuum hence induces suction. In order to verify this newly developed system, mechanical properties and suction distribution influenced by the new artificial root and selected living tree were tested and compared in an atmospheric controlled room. The comparisons of test results showed that the suction distributions induced by the artificial root and the living tree were reasonably close, during both transpiration and a rainfall event.
\end{abstract}

Keywords: Physical modelling, Plant transpiration, Suction, Unsaturated soils,

\section{INTRODUCTION}

Vegetation has been recognized to be a sustainable alternative for improving shallow slope instability. Extensive research has demonstrated the use of mechanical root reinforcement to enhance slope stability (Wu et al. 1988; Greenwood et al. 2004, among others). The mechanical root reinforcement was also investigated in slope model tests reinforced with rigid or flexible artificial roots in centrifuge (Sonnenberg et al. 2012). This modelling technique enables reduced scaled models to be tested at the stress levels identical to field experiments but much more controlled test conditions. However, any effects of suction induced by plant transpiration on slope stability are generally ignored in existing artificial roots. It is well-recognised that an increase in soil suction would increase soil shear strength (Ng and Menzies 2007) and also decrease water permeability (Ng and Leung 2012). Limited field (Simon and Collison 2002, among others) and laboratory studies ( $\mathrm{Ng}$ et al. 2013, among others) have shown that vegetated soil could retain suction ranging from $5-10 \mathrm{kPa}$ within root zone after $10-100$ years return period of rainfall. More investigations are needed to investigate the hydrological effect of plants on induced soil suction and slope stability during rainfall condition, which engineers concern most.

This paper aims to develop and test a simplified, yet robust artificial root that can simulate the effects of plant transpiration in physical model tests including centrifuge. Scaled mechanical and hydrological properties of a material that was used to produce the artificial root were verified by comparing with those of living tree roots. Then, suction distribution created by the artificial root were measured and compared with those induced by the living tree in laboratory under $1 \mathrm{~g}$ condition.

\section{WORKING PRINCIPLE OF ARTIFICIAL ROOT}

\subsection{Simulation of plant root water-uptake}

Fig. 1(a) shows the simplified process of plant root water-uptake. Upward movement of water is actively regulated by evapotranspiration of leaf and stomata (Holbrook et al. 1995; Pockman et al. 1995). The water flow is therefore driven by difference in hydraulic head inside the root xylem. The artificial root system intends to capture and simulate this process. It was designed in such a way that the hydraulic head inside the artificial 
root could be lowered by vacuum being supplied and controlled in a vacuum chamber (Kamchoom et al. 2014; $\mathrm{Ng}$ et al. 2014). The artificial root was made of a high air-entry value (AEV) filter. Because of the small, uniform pore sizes of this kind of filter, the negative water pressure generated by the vacuum can be maintained in the water-saturated filter, as long as the applied vacuum is less than the AEV of the filter. Since the porous filter is in contact with soil, the lower head inside the artificial root would result in upward water flow from the soil to the chamber through the filter. This would reduce soil moisture and hence induce soil suction. In this study, cellulose acetate (CA) is selected to be the porous filter (see Fig. 1(b)) because it has considerably high AEV of up to $100 \mathrm{kPa}$. Thus, the hydraulic gradient produced by any applied vacuum (i.e., < $-98 \mathrm{kPa}$ ) in the chamber can be maintained.

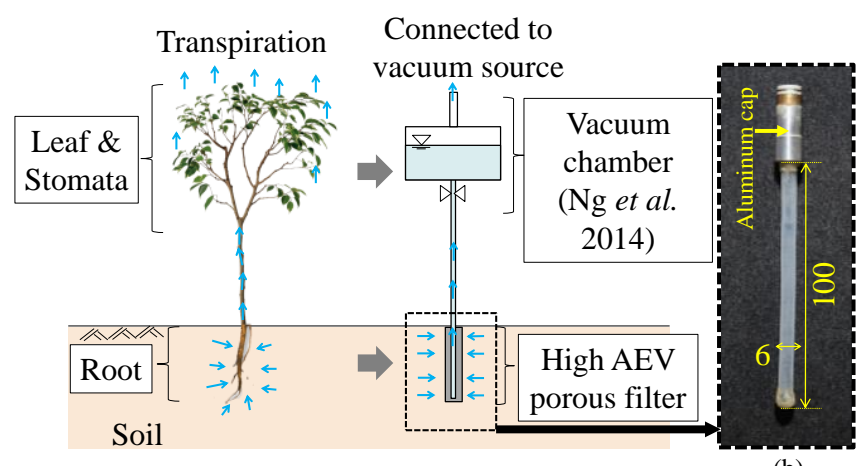

(a)

(b)

Fig. 1. (a) Idealisation of plant root water-uptake and overview of (b) artificial root made of cellulose acetate filter. All dimensions are in millimeters

\subsection{Mechanical properties of artificial root}

In addition to simulating the hydrological effect of transpiration-induced suction, the CA has scaled mechanical properties that are similar to those of living plant roots. The filter is straight in design and it is a representation of taproot architecture that is broadly categorized by Fitter and Stickland (1991) and Stokes and Mattheck (1996). Such taproot is thus suitable for representing some willow (Salix) species (Kuzovkina and Volk 2009) and Schefflera heptaphylla whose roots are identified to be taproot-shaped (Leung 2014). This species was selected for investigation because it is commonly found in many parts of Asia such as Hong Kong, Japan and Thailand for ecological restoration and slope bioengineering. The filter is a long circular hollow tube with inner and outer diameters of 4 and 6 $\mathrm{mm}$, respectively. The CA was cut to $110 \mathrm{~mm}$ long, while $10 \mathrm{~mm}$ of it was inserted into an aluminum cap that can facilitate the connection to the vacuum system. Any void between the CA and the cap was sealed with epoxy. At the other end of the CA, it was plugged with rubber and again sealed with epoxy. As a result, the total root depth is $100 \mathrm{~mm}$.

$\mathrm{CA}$ has an elastic modulus (E) of $83 \mathrm{MPa}$ and a tensile strength $\left(\sigma_{T}\right)$ of $31 \mathrm{MPa}$, both of which are fairly close to those typically identified in real roots (Stokes and Mattheck 1996). Kamchoom et al. (2014) carried out a series of direct shear tests under different normal stresses to measure soil-root interface friction. Both the peak and ultimate interface friction angles are $34^{\circ}$, which is close to the typical range $\left(36.1^{\circ}-37.9^{\circ}\right)$ for soil-real root interface (Wang et al., 2010). In this study, the axial rigidity (EA) of each artificial root was scaled instead of flexural rigidity (EI). This is because root reinforcement against pull-out and slope failure was mainly contributed by the mobilization of axial strain of roots (Sonnenberg et al. 2012; Kamchoom et al. 2014). Scaling factor of EA is $1 / \mathrm{N}^{2}$ where the model subjected to $\mathrm{N}$ times of a "gravitational" acceleration (Taylor 1995). The dimension of artificial root in this paper was scaled for target $15-\mathrm{g}$. The prototype EA is thus $290 \mathrm{kPa}-\mathrm{m}^{2}$. Fig. 2 compares the prototype EA of different materials that have been selected for making artificial roots and Schefflera heptaphylla. While the EA of wood and rubber provide an upper and lower bounds, CA is found to be within the bounds and represents the average value of the tree.

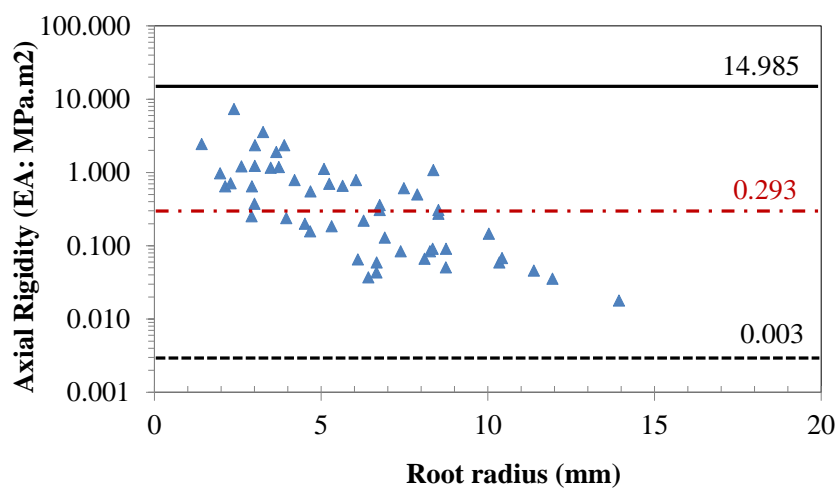

$\Delta$ Schefflera heptaphylla (Leung 2014)

----Viton rubber (Sonnenberg et al. 2012)

— Linden wood (Sonnenberg et al. 2012)

-.CA (this study)

Fig. 2. Comparison of axial rigidity (EA) of real root with artificial root made of different materials

\section{VERIFICATION OF ARTIFICIAL ROOT AGAINST LIVING TREE}

\subsection{Test plan}

In this study, three laboratory tests were carried out under a controlled atmospheric condition $(\mathrm{Ng}$ et al. 2013). In the first test, distribution of soil suction induced by the artificial root "transpiration" was measured in a test box (denoted as Test M). In the second test, a living tree was transplanted in a separate test box with an identical condition as Test $\mathbf{M}$ for comparing suction induced by transpiration (denoted as Test $\mathrm{T}$ ). The last test was a control experiment, in 
which any suction induced in a separate bare test box (i.e., without installing artificial root or vegetating living tree; denoted as Test B) was measured.

\subsection{Experimental setup and instrumentation}

A typical setup is shown in Fig. 3. Each test box was a rigid rectangular with a square cross section (300 $\mathrm{mm} \times 300 \mathrm{~mm}$ ) There were nine numbers of $5 \mathrm{~mm}$ holes located at the bottom of each box for free drainage. The soil tested in this study is completely decomposed granite (CDG), which is commonly found in Hong Kong. In all three tests, the CDG was compacted in each test box by the undercompaction method (Ladd, 1977) to $280 \mathrm{~mm}$ depth. Some measured properties of the CDG are summarised in Table 1.

Table 1. Properties of CDG

\begin{tabular}{ll}
\hline Index of CDG & value \\
\hline Specific gravity & 2.6 \\
\hline Maximum dry density $(\mathrm{kg} / \mathrm{m} 3)$ & 1870 \\
\hline Optimum moisture content $(\%)$ & 13 \\
\hline Gravel content $(>2 \mathrm{~mm}, \%)$ & 19 \\
\hline Sand content $(\leq 2 \mathrm{~mm}, \%)$ & 42 \\
\hline Silt content $(\leq 63 \mu \mathrm{m}, \%)$ & 27 \\
\hline Clay content $(\leq 2 \mu \mathrm{m}, \%)$ & 12 \\
\hline Coefficient of uniformity & 9.4 \\
\hline Coefficient of curvature & 3.3 \\
\hline Plastic limit $(\%)$ & 26 \\
\hline Liquid limit $(\%)$ & 44 \\
\hline Plasticity index $(\%)$ & 18 \\
\hline USCS & Silty sand $(\mathrm{SM})$ \\
\hline Dry density $(\mathrm{kg} / \mathrm{m} 3)$ & 1496 \\
\hline Gravimetric water content $(\%)$ & 12 \\
\hline Degree of compaction $(\%)$ & $80 \pm 2$ \\
\hline
\end{tabular}

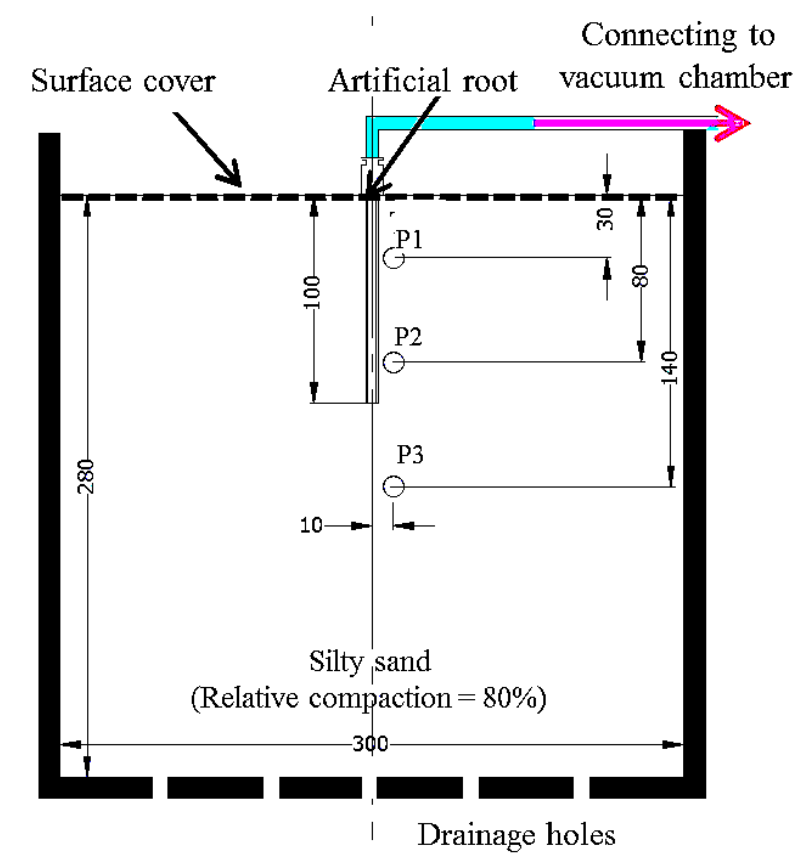

Fig. 3. Typical test setup for artificial root (Test M) and living tree (test T). All dimensions are in millimeters

In order to provide better hydraulic contact between the artificial root and the surrounding soil, a hole was drilled into a smaller diameter than of artificial root at the center of the box for Test M. For Test T, a living tree species Schefflera heptaphylla, which has an average root length of $104 \mathrm{~mm}$, was transplanted to the center of a separate box. Procedures of plantation were reported in $\mathrm{Ng}$ et al. (2013).

Regarding instrumentation, three miniature-tip tensiometers, namely $\mathrm{P} 1, \mathrm{P} 2$, and $\mathrm{P} 3$, were installed at $10 \mathrm{~mm}$ next to the centerline of each box to measure vertical distribution of negative pore water pressure (PWP). The measurement depths were 30, 80, and 140 mm depths.

\subsection{Test procedures}

Before testing, a similar suction profile was achieved in the three boxes by applying identical cycles of surface ponding and evaporation, as detailed in $\mathrm{Ng}$ et al. (2013). Then, the boxes for Tests $\mathrm{M}$ and $\mathrm{T}$ were subjected to two-stage of testing in an atmospheric -controlled room. In the first stage, a constant vacuum pressure of $-98 \mathrm{kPa}$ (upper limit of the vacuum system) was maintained through the artificial root in Test $M$ for 48 hours. Any changes of suction in each test were monitored by the three tensiometers (see Fig. 3). In order to minimize any suction induced by evaporation, the soil surface was covered with plastic sheets. Any measured suction change was thus attributed to applied vacuum in Test $M$ and tree transpiration in Test $T$

At the end of drying period, the second stage of test was to apply a rainfall event to each box. The rainfall intensity and duration were $333 \mathrm{~mm} / \mathrm{hr}$ and 2 minute, respectively. This represented a rainfall event with a return period of 100 years, according to statistical analysis of 100 years' rainfall data in Hong Kong conducted by Lam and Leung (1995). During the rainfall event, the plastic sheet cover was temporarily removed. When the rainfall ceased, the soil surface was covered again for suction to be equilibrated until all tensiometers showed less than $1 \mathrm{kPa}$ (i.e., accuracy of each tensiometers) change in PWP. For the entire second stage, the vacuum pressure was maintained at $95 \mathrm{kPa}$ in Test $\mathrm{T}$. Note that for Test B, only the first stage of test was performed.

\section{INTERPRETATION OF TEST RESULTS}

\subsection{Variations of induced soil suction with time}

The measured variations of PWP from Tests $\mathrm{M}, \mathrm{T}$ and $\mathrm{B}$ are compared in Fig. 4. For Test B, suction was found to slightly increase by less than $2 \mathrm{kPa}$ due to downward flow towards the bottom drainage holes. When the living tree was present in Test $\mathrm{T}$, suction induced by transpiration is found to increase at a decreasing rate, and more than those observed in Test B. After transpiring for 48 hours, the peak induced suction was $25 \mathrm{kPa}$ at $30 \mathrm{~mm}$ depth (see Fig. 4(a)) and about $4 \mathrm{kPa}$ at $140 \mathrm{~mm}$ depth (see Fig. 4(b)). For Test 
$\mathrm{M}$, the measurement showed that the vacuum operation of artificial root was capable of inducing significant amount of suction at $30 \mathrm{~mm}$ depth (Fig. 4(a)). There was a similar trend of variation of suction between Tests $\mathrm{M}$ and $\mathrm{T}$ during drying period, though the suctions induced by the artificial root (i.e., $19 \mathrm{kPa}$ ) was slightly lower by about $5 \mathrm{kPa}$. At the deeper depth of $140 \mathrm{~mm}$ (Fig. 4(b)), the response of suction in Test M was insensitive to the vacuum operation as well as transpiration in Test $\mathrm{T}$.

After rainfall, the suction retained at $30 \mathrm{~mm}$ depth (Fig. 4 (a)) was $2 \mathrm{kPa}$ in both Tests $\mathrm{M}$ and $\mathrm{T}$ until the end of the test. This is likely attributed to the effects of the applied vacuum in Test $M$ and tree transpiration in Test T. While the soil within the root zone was subjected to the suction decrease due to water infiltration, the water uptake process generated by the artificial root and that induced by tree transpiration help maintained certain amount of suction. On the other hand, the suction response at $140 \mathrm{~mm}$ depth (Fig. 4(b)) is found to be similar between Tests $\mathrm{M}$ and $\mathrm{T}$, though the suction at this particular depth was reduced to zero.

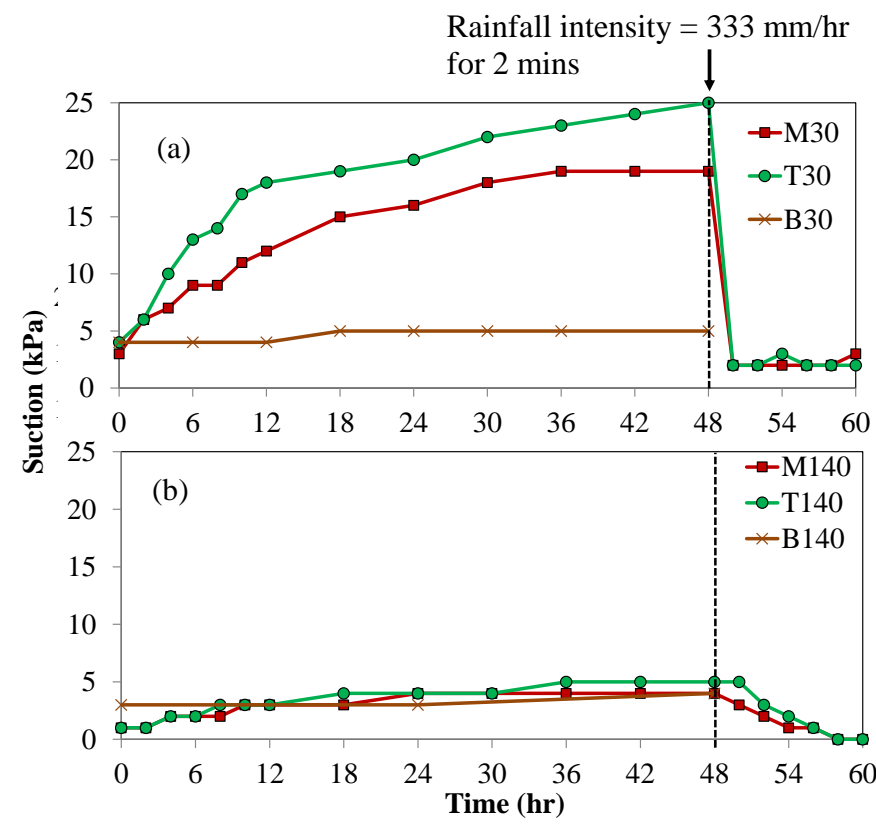

Fig. 4. Measured variations of suction with time (a) within root depth (at $30 \mathrm{~mm}$ depth) and (b) beyond root depth $(140 \mathrm{~mm}$ depth)

\subsection{Observed vertical distribution of induced suction}

Measured suction profiles of Tests $\mathrm{M}$ and $\mathrm{T}$ during drying period are shown in Fig. 5(a). It can be seen that the initial suction profiles in Test $\mathrm{M}$ and $\mathrm{T}$ are close to each other. After the first stage of test, suction increased substantially within the root depth due to tree transpiration in Test $\mathrm{T}$ and the applied vacuum pressure in Test M. The induced suction at 30 and $80 \mathrm{~mm}$ depth was uniform for both tests. This is because the artificial root induced the same amount of vacuum throughout the root depth, and thus the soil at both 30 and $80 \mathrm{~mm}$ depths were subjected to similar hydraulic gradient for inducing similar amount of suction. Similarly, a tap-shaped root of tree has uniform root area index (RAI) along the root depth and this influenced uniform induced suction within the depth (Garg et al. 2015). On the contrary, the induced suction at $140 \mathrm{~mm}$ depth was less than those observed within root depth, because this depth was further (i.e., $40 \mathrm{~mm}$ ) away from the root depth and thus the hydraulic gradient was relatively lower. This suggests that the influence depth of suction due to both the tree transpiration and the applied vacuum was deeper than the root depth.
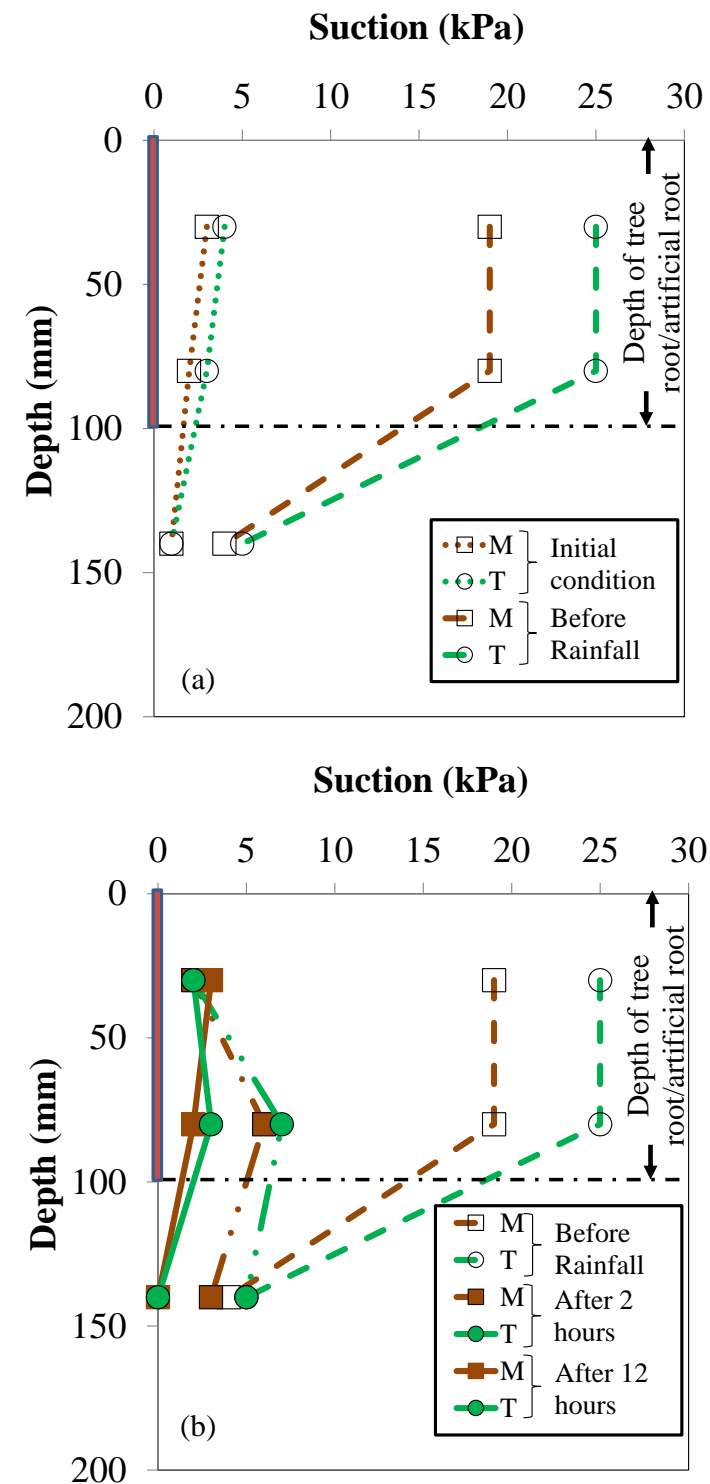

Fig. 5. Comparisons of suction profiles influenced by artificial root (Test $\mathrm{M}$ ) and living tree (Test $\mathrm{T}$ ) during (a) drying and (b) after rain period

After 2 hours of rainfall event (Fig. 5(b)), suction at the shallowest depth of $30 \mathrm{~mm}$ was dropped significantly. However, suction in deeper depths can be retained. The drop of suction in Test $\mathrm{M}$ was more than those in Test $\mathrm{T}$. This is because the initial suction 
before rainfall in Test $\mathrm{M}$ was lower than that in Test $\mathrm{T}$ and thus the soil in Test $\mathrm{M}$ had a higher water permeability for rainwater to infiltrate to deeper depths. At the end of the test, suction was reduced further and equilibrated at a similar suction of about 2 to $3 \mathrm{kPa}$ for 30 and $80 \mathrm{~mm}$ depth. This demonstrates the effectiveness of utilizing the new artificial roots to re-create the suction induced/retained by the living tree.

\section{SUMMARY AND CONCLUSION}

A new artificial root that can simulate the effects of transpiration and its induced suction is developed and verified in this study. The artificial root is tap-shaped and is designed to represent some tree species commonly used for ecological restoration and slope bioengineering. The artificial root consists of a high air-entry value porous filter, cellulose acetate, which has scaled mechanical properties similar to real roots. The porous filter is in contact with soil and it is connected to vacuum system. The function of the high AEV filter, which is fully-saturated with de-aired water, is to maintain hydraulic gradient between soil and the root system. The lower hydraulic head due to vacuum inside the artificial root would lead to water flow from soil to the root. Any soil moisture loss would hence induce suction in soil.

This study shows that the artificial root was capable of simulating the effects of tree transpiration and hence producing credible suction that can be induced by a living tree, Schefflera heptaphylla, during both drying period and a rainfall event with a return period of 100 years The design of such tap-shaped artificial root provides a simple yet robust tool for researchers and engineers to investigate the influence of suction induced by roots on geotechnical problems such as the stability of slopes and evapotranspirative landfill covers in centrifuge model tests at high-g condition. Further modification is currently underway for modelling plants that have different root geometries, such as heart and plate shapes.

\section{ACKNOWLEDGEMENTS}

A research grant (HKUST6/CRF/12R) provided by the Research Grants Council of the Government of the Hong Kong SAR and another one (2012CB719805) provided by the Ministry of Science and Technology of the People's Republic of China under the National Basic Research Program (973 Program) are acknowledged. The second author would also like to acknowledge the EU Marie Curie Fellowship provided under the Career Integration Grant for the project "BioEPIC", and research travel support from the Northern Research Partnership.

\section{REFERENCES}

1) Fitter, A. H. and Stickland, T. R. (1991): Architectural analysis of plant root systems, New phytologist, 118(3), $383-$ 389.

2) Garg, A., Leung, A. K. and Ng, C. W. W. Comparisons of suction induced by evapotranspiration and transpiration of $\mathrm{S}$. heptaphylla. Under review in Canadian Geotechnical Journal.

3) Greenwood, J. R., Norris, J. E. and Wint, J. (2004): Assessing the contribution of vegetation to slope stability, Geotechnical Engineering, 157(4), 199-207.

4) Holbrook, N. M., Burns, M. J., and Field, C. B. (1995): Negative xylem pressures in plants: a test of the balancing pressure technique, Science New York then Washington, 1193-1193.

5) Kamchoom, V., Leung, A. K. and Ng, C. W. W. (2014): Effects of root geometry and transpiration on pull-out resistance, Geotechnique Letters, 4(1), 330-336.

6) Kuzovkina, Y. A., and Volk, T. A. (2009): The characterization of willow (Salix L.) varieties for use in ecological engineering applications: Co-ordination of structure, function and autecology, Ecological Engineering, 35(8), 1178-1189.

7) Ladd, R. S. (1977): Specimen preparation and cyclic stability of sands, Journal of Geotechnical Engineering Division, 103(6), 535-547.

8) Lam, C. C. and Leung, Y. K. (1995): Extreme rainfall statistics and design rainstorm profiles at selected locations in Hong Kong. Hong Kong: Royal Observatory.

9) Leung, T. Y. (2014): The use of native woody plants in slope upgrading in Hong Kong, PhD Thesis, The University of Hong Kong, Hong Kong.

10) Ng, C. W. W. and Leung, A. K. (2012): Measurements of drying and wetting permeability functions using a new stress-controllable soil column, Journal of Geotechnical and Geoenvironmental Engineering, 138(1), 58 - 68.

11) Ng, C. W. W., Leung, A. K., Kamchoom, V. and Garg, A. (2014): A Novel Root System for Simulating Transpiration-Induced Soil Suction in Centrifuge. Geotechnical Testing Journal, 37(5), 1-15.

12) Ng, C. W. W. and Menzies, B. (2007): Advanced Unsaturated Soil Mechanics and Engineering, Taylor and Francis, London, 687, ISBN: 978-0-415-43679-3.

13) Ng, C. W. W., Woon, K. C., Leung, A. K. and Chu, L. M. (2013): Experimental investigation of induced suction distribution in a grass-covered soil, Ecological Engineering, 52(1), $219-223$.

14) Pockman, W. T., Sperry, J. S., and O'Leary, J. W. (1995): Sustained and significant negative water pressure in xylem. Nature, 378(6558), 715-716.

15) Simon, A. and Collison, A (2002): Quantifying the mechanical and hydrologic effects of riparian vegetation on strembank stability, Earth Surface Process Landform, 27(1), $527-546$.

16) Sonnenberg, R., Bransby, M. F., Bengough, A. G., Hallett, P. D. and Davies, M. C. R. (2012): Centrifuge modelling of soil slopes containing model plant roots, Canadian Geotechnical Journal, 49(1), 117.

17) Stokes, A. and Mattheck, C. (1996): Variation of wood strength in tree roots, Journal of Experimental Botany, 47(5), $693-699$.

18) Taylor, R. N. (1995): Geotechnical Centrifuge Technology, Taylor and Francis.

19) Wu, T. H., Beal, P. E. and Lan, C. (1988): In-situ shear test of soil-root systems, Journal of Geotechnical Engineering, 114(1), 1376-1394. 